\title{
COMPUTATIONAL SIMULATION OF FULLY TRIMMED FLIGHT OF A HELICOPTER IN HOVER
}

\author{
Wieńczysław Stalewski, Wiesław Zalewski, Katarzyna Surmacz \\ Institute of Aviation \\ Krakowska Av. 110/114, 02-256 Warszawa, Poland \\ tel.: +48228460011 fax: +48943426753 \\ e-mail:wienczyslaw.stalewski@ilot.edu.pl,wieslaw.zalewski@ilot.edu.pl \\ katarzyna.surmacz@ilot.edu.pl \\ Maximilian Pulfer, Frieder Hirsch \\ Kopter Germany GmbH \\ e-mail:maximilian.pulfer@koptergroup.de,frieder.hirsch@koptergroup.de
}

\begin{abstract}
In trimmed flight of a helicopter, all the forces and moments, aerodynamic, inertial, and gravitational, are in balance. Keeping the helicopter in trimmed state, needs a precise adjustment of flight controls. The methodology of simulation of a fully trimmed flight of rotorcraft has been developed and applied to simulate hover of a helicopter.

The presented approach is based on a solution of Unsteady Reynolds-Averaged Navier-Stokes (URANS) equations. In contrast to typical solutions of such problem, in the newly developed methodology, the flight controls corresponding to the trimmed-flight conditions are also determined based on the solution of URANS equations.

The methodology is based on coupling of several computational models of Computational Fluid Dynamics and Flight Dynamic. The URANS equations are solved in a three-dimensional region surrounding the flying helicopter, using the ANSYS FLUENT code. The approach is truly three-dimensional, with truly modelled geometry and kinematics of main and tail rotor blades. This applies to modelling of blade flapping and lead-lag motion, too.

The trimming procedure uses six independent parameters (i.e. collective and cyclic pitch of main rotor blades, collective pitch of tail rotor blades, pitch, and bank angles of a helicopter) that should be adjusted so as to balance all forces and moments acting on the helicopter.

The detailed description of the developed methodology as well as the results of simulation of trimmed hover of the helicopter was presented.
\end{abstract}

Keywords: helicopter flight simulation, hover, trimmed flight, URANS

\section{Introduction}

In trimmed flight of a helicopter all the forces, aerodynamic, inertial, and gravitational, as well as the overall moment vectors are in balance. Keeping the helicopter in trimmed state, needs a precise adjustment of flight controls (i.e. collective and cyclic pitch controls of main rotor blades, collective pitch control of tail rotor blades, etc.) [2]. Computational simulation of such flight state is a very challenging task, especially when flight simulation is conducted using the Navier-StokesEquations solver to determine precisely all interactions between the air and helicopter surface. Usually, in such approach, the helicopter flight controls corresponding to the trimmed flight are determined using simplified aerodynamic models of rotors (e.g. Blade Element Theory) and other components of a helicopter (e.g. tabulated, static aerodynamic characteristics of a fuselage, stabilizers, vertical fin, etc.) [3, 4]. Then, these determined in simplified manner flight controls are used in an advanced flight simulator based on the Navier-Stokes Equations. However, this approach can in fact lead to flight states significantly distant from a trimmed flight. The reason may be incompatibilities between simplified and advanced aerodynamic models of a helicopter flight.

The presented research focused on development of methodology of simulation of trimmed flight of a helicopter based on a solution of Unsteady Reynolds-Averaged Navier-Stokes (URANS) 
equations. In contrast to the approach described above, in the newly developed methodology, the flight controls corresponding to the trimmed-flight conditions are also determined by the solution of URANS equations.

\section{Methodology}

A flight of a helicopter has been simulated based on computational methodology schematically presented in Fig. 1. The flight simulation consists in the solution of URANS equations in the domain surrounding the helicopter. The URANS equations are solved using the ANSYS FLUENT [1] code. All specific rotorcraft activities are realised by developed in-house UDF-module Virtual-Rotor-3D. This module is responsible for modelling of rotation of the main and tail rotors, blade feathering, and flap-and-lag motion that is determined independently for each blade of main rotor through a solution of ordinary differential equations simultaneously with the solution of URANS equations. The structure of computational mesh is shown in Fig. 2 and Fig. 3. The motion of main-rotor blades is modelled by the Overset Method (computational-mesh overlapping) while the tail-rotor-blade motion is modelled based on Sliding Mesh Method.

In the presented approach, the helicopter-flight control is conducted using the following parameters:

- collective pitch of main-rotor blades: $\quad \theta_{0}$,

- components of cyclic pitch of main-rotor blades: $\theta_{\mathrm{S}}, \theta_{\mathrm{C}}$,

- collective pitch of tail-rotor blades: $\quad \theta_{\mathrm{TR}}$,

- pitch and bank angles of a helicopter $\varphi, \phi$.

The above parameters may be changed smoothly during the simulation of helicopter flight, which is used when trimming the helicopter. In the presented approach, the helicopter trimming procedure consists in establishing the flight-control vector: $\bar{\Theta}=\left[\theta_{0}, \theta_{S}, \theta_{C}, \theta_{T R}, \varphi, \phi\right]$, so as to obtain required dimensionless forces and moments: $\bar{T}=\left(C_{L-W}, C_{D}, C_{S}, C_{l}, C_{m}, C_{n}\right)$ acting on the helicopter, where:

- $\mathrm{C}_{\mathrm{L}-\mathrm{W}}$ - lift-weight-imbalance coefficient, (dimensionless difference between the resultant lift force acting on the helicopter and the helicopter weight),

- $\mathrm{C}_{\mathrm{D}}-$ drag coefficient,

$-\mathrm{C}_{\mathrm{S}}$ - side force coefficient,

$-\mathrm{C}_{1}$ - rolling-moment coefficient,

$-\mathrm{C}_{\mathrm{m}}$ - pitching moment coefficient,

$-\mathrm{C}_{\mathrm{n}}$ - yawing moment coefficient.

The moment reference point coincides with the centre of gravity of the helicopter. The balance of side forces acting on the helicopter has been omitted in the presented research. For given flight priority, the required values of $C_{L-W}, C_{D}, C_{l}, C_{m}, C_{n}$ are typically set as follows: $C_{L-W}=C_{D}=C_{S}=$ $\mathrm{C}_{\mathrm{l}}=\mathrm{C}_{\mathrm{m}}=\mathrm{C}_{\mathrm{n}}=0$ which means fully trimmed state of a helicopter flight.

The problem is defined in mathematical terms as minimisation of function:

$$
\Phi(\bar{\Theta})=\sqrt{\mathrm{C}_{\mathrm{L}-\mathrm{W}}{ }^{2}+\mathrm{C}_{\mathrm{D}}{ }^{2}+\mathrm{C}_{\mathrm{S}}{ }^{2}+\mathrm{C}_{\mathrm{l}}^{2}+\mathrm{C}_{\mathrm{m}}{ }^{2}+\mathrm{C}_{\mathrm{n}}{ }^{2}} .
$$

The applied trimming procedure is based on classic, iterative approach. The new, corrected values of flight-control vector $\bar{\Theta}_{n}$ are evaluated according to the formula:

$$
\bar{\Theta}_{\mathrm{n}}=\bar{\Theta}_{\mathrm{p}}+\left[\frac{\partial \overline{\mathrm{T}}}{\partial \overline{\mathrm{\Theta}}}\right]^{-1} \cdot\left(\overline{\mathrm{T}}_{\mathrm{r}}-\overline{\mathrm{T}}_{\mathrm{p}}\right)
$$

where: $\bar{\Theta}_{p}$ is the current value of control vector $\bar{\Theta}, \overline{\mathrm{T}}_{p}$ is the current value of force-moment vector $\overline{\mathrm{T}}, \overline{\mathrm{T}}_{r}$ is the required value of vector $\overline{\mathrm{T}}$, and $\left[\frac{\partial \overline{\mathrm{T}}}{\partial \bar{\Theta}}\right]$ is the gradient matrix. Usually, to obtain relatively 
good convergence of helicopter trimming, the procedure (2) has to be applied several times, iteratively.

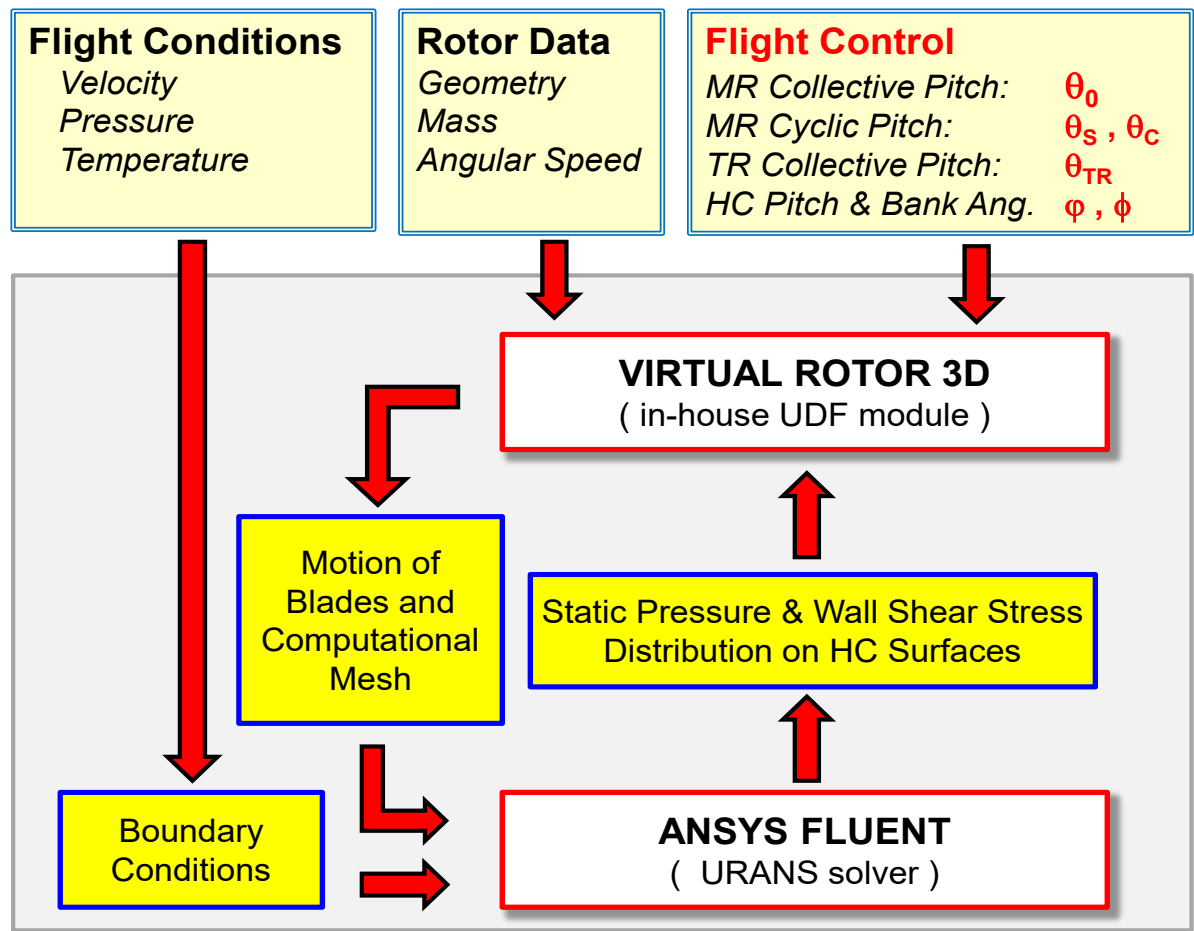

Fig. 1. The general scheme of developed methodology of simulation of trimmed flight of helicopter

Quality of evaluation of the gradient matrix $\left[\frac{\partial \bar{T}}{\partial \overline{\bar{\theta}}}\right]$ strongly influences the success of the helicoptertrimming process. In the presented approach, the gradient matrix is evaluated using the Least Mean Square method, based on results of helicopter-flight simulations conducted several times for different values of flight-control-parameter vector $\bar{\Theta}$. In general, the gradient matrix $\left[\frac{\partial \overline{\mathrm{T}}}{\partial \overline{\mathrm{\Theta}}}\right]$ should be evaluated/ modified in each iterative step of the trimming process and for each computational case of a helicopter flight.

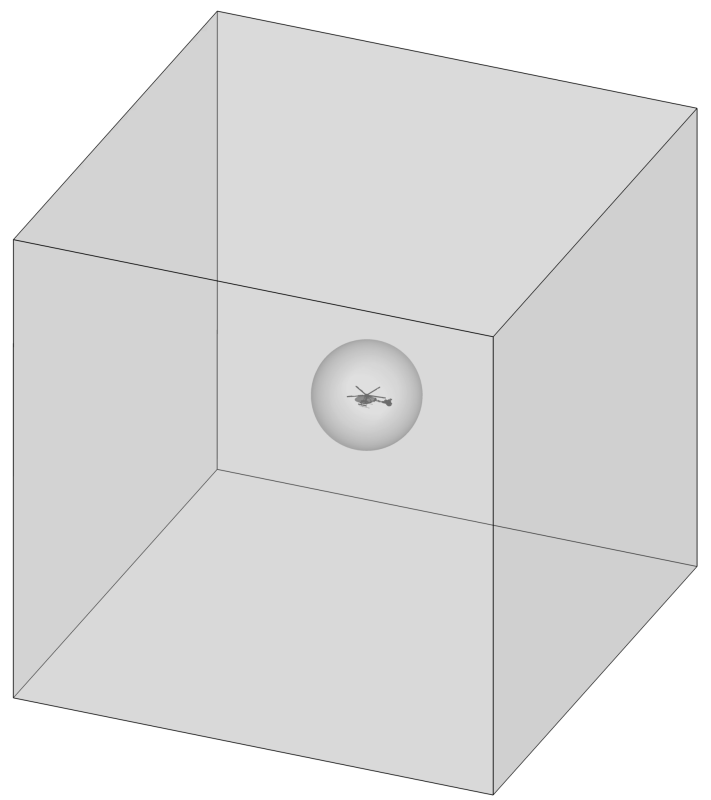

Fig. 2. Structure of computational domain 


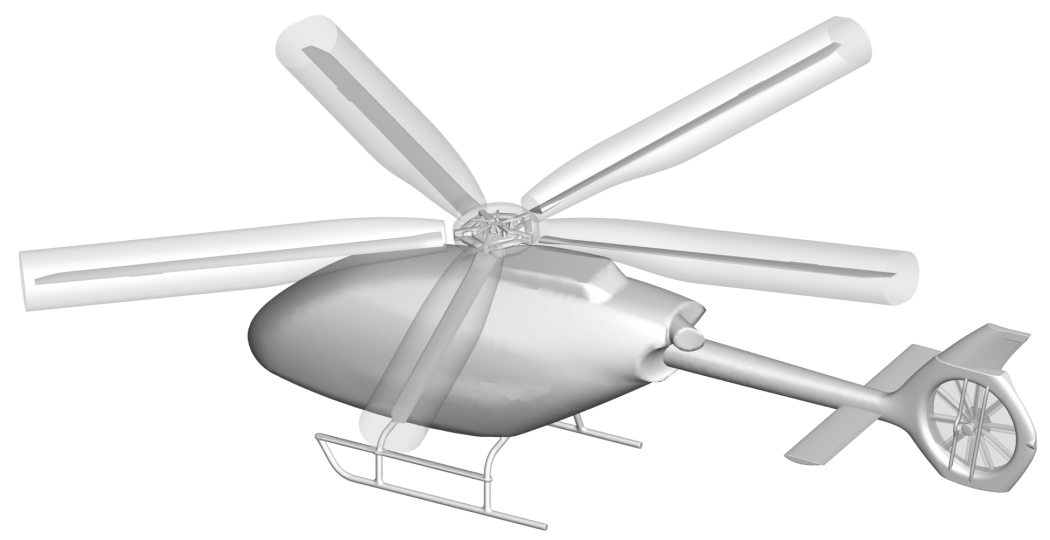

Fig. 3. Local computational domains around main rotor blades used in trimmed-flight simulations

\section{Results of simulations of a trimmed flight of a helicopter}

The simulations of trimmed flight have been conducted for the computational model of the helicopter presented in Fig. 4. The flight has been simulated at the altitude $400 \mathrm{~m}$ in standard ISA atmospheric conditions.

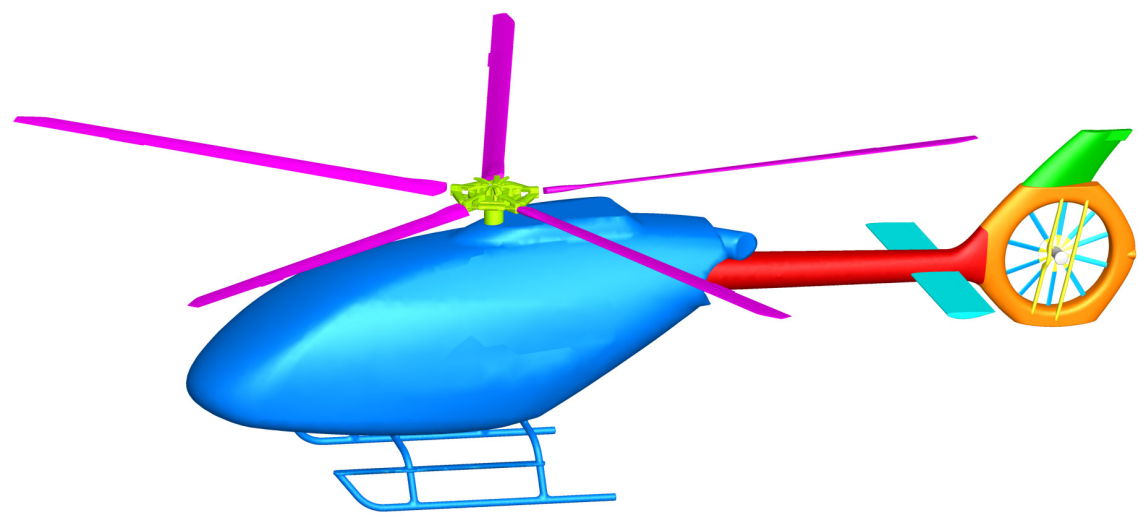

Fig. 4. Computational model of the helicopter used in simulations of trimmed flight

In initial stage of the research, several auxiliary helicopter-flight simulations have been conducted to obtain the data necessary to determine the gradient matrix required for the trimming procedure. Next, the actual simulation of flight in hover (without a ground effect) has been conducted. Initially, the flight controls have been established based on simplified evaluations. Based on these settings, the helicopter-flight simulation has been conducted, using the ANSYS FLUENT solver together with then Virtual-Rotor-3D UDF module. After reaching satisfactory convergence and periodicity of flow, the value of current force-and-moment vector $\bar{T}=\left(C_{L-W}, C_{D}, C_{S}, C_{l}, C_{m}, C_{n}\right)$ has been evaluated based on forces and moments averaged during one revolution of main rotor. Next, using the formula (2), a new corrected value of flightcontrol vector $\bar{\Theta}_{n}$ has been determined. The helicopter flight simulation has been repeated for the corrected settings of flight controls. After this process, the new state of the helicopter flight has been obtained. In general, this new flight state should be much more balanced than the initially investigated flight of helicopter in hover.

Table 1 shows the percentage measure of balance of dimensionless forces and moments acting on the helicopter in relation to their values before trimming. In the table, a value $100 \%$ means fully balanced given component of force or moment, while $0 \%$ means absence of any change of force or moment before and after trimming. In the discussed case, one can observe that trimming procedure 
has been especially effective in a case of balancing of forces acting on the helicopter. In the case of rolling and pitching moment, the improvement of balance is also satisfactory. However, in the case of yawing moment coefficient $\left(\mathrm{C}_{n}\right)$, there is a negative percentage, which means that after trimming, the imbalance of this moment component is even worse than before trimming.

Such result does not mean that the described trimming procedure has failed in the discussed case. From point of view of the minimisation of function $\Phi$ defined in (1), the procedure has significantly improved the balance of forces and moments acting on the helicopter. While for initially assumed flight controls the value of this function was $\Phi(\bar{\Theta})=6.98445 \cdot 10^{-4}$, the value of $\Phi$ for the flight controls determined by the trimming procedure was significantly reduced to $\Phi(\bar{\Theta})=1.05316 \cdot 10^{-4}$. This means nearly sevenfold improvement in the balance of forces and moments after conducting of the trimming procedure.

Tab. 1. Percentage measure of full-balance of dimensionless forces and moments in relation to their values before trimming

\begin{tabular}{|l|c|c|c|c|c|c|}
\hline Coefficient & $\mathrm{C}_{\mathrm{L}-\mathrm{W}}$ & $\mathrm{C}_{\mathrm{D}}$ & $\mathrm{Cs}$ & $\mathrm{C}_{1}$ & $\mathrm{C}_{\mathrm{m}}$ & $\mathrm{C}_{\mathrm{n}}$ \\
\hline Full-Balance Measure & $93.3 \%$ & $99.0 \%$ & $99.9 \%$ & $52.5 \%$ & $70.5 \%$ & $-79.4 \%$ \\
\hline
\end{tabular}

Figure 5 and 6 presents results of trimming obtained for the discussed case of hover of a helicopter. Top graphs in the figures present time-dependent resultant forces or moment acting on the helicopter, captured during one period of revolution of the main rotor, before conducting of the trimming. Bottom graphs in the figures show the same parameters measured after finalizing the trimming procedure.
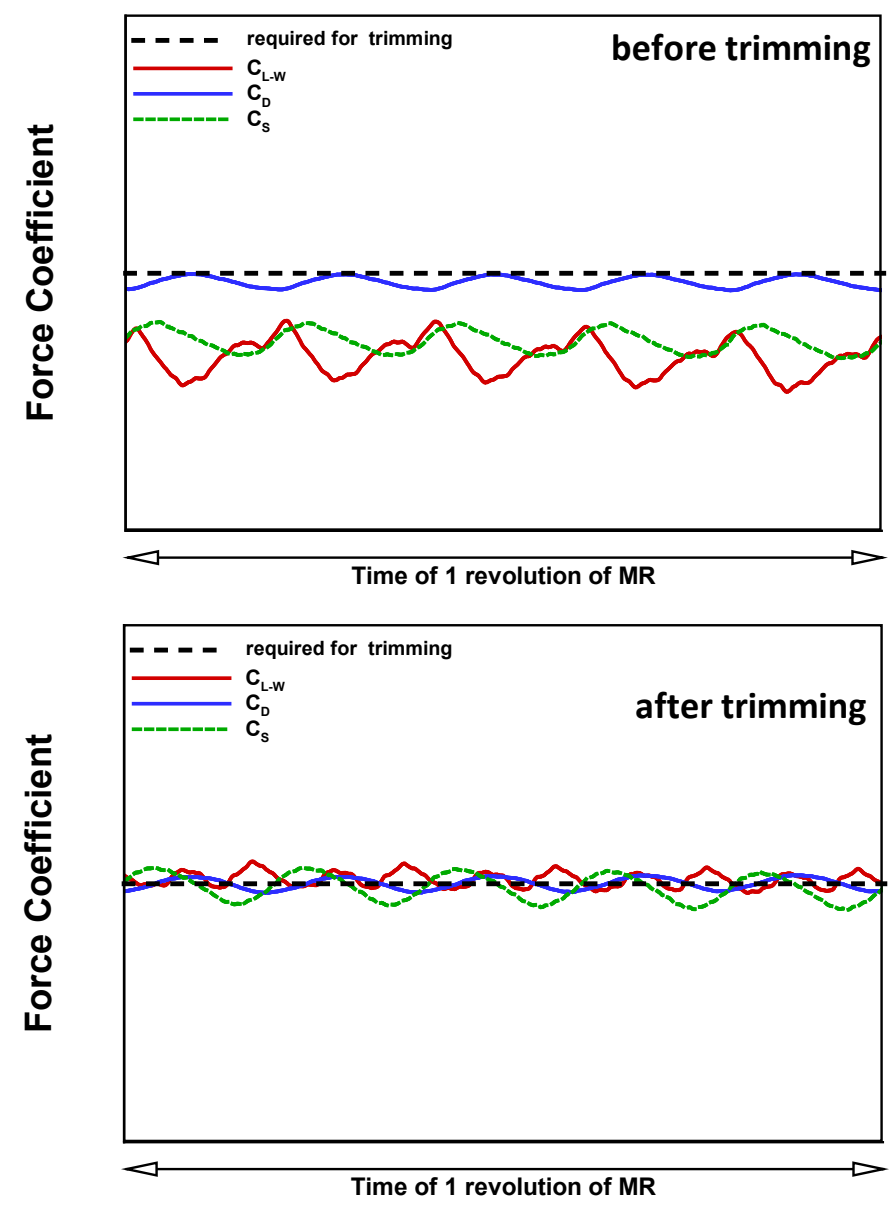

Fig. 5. Coefficients of global forces $\left(C_{L-W}, C_{D}, C_{S}\right)$ acting on the helicopter in hover vs. time, before (top) and after (bottom) trimming procedure 

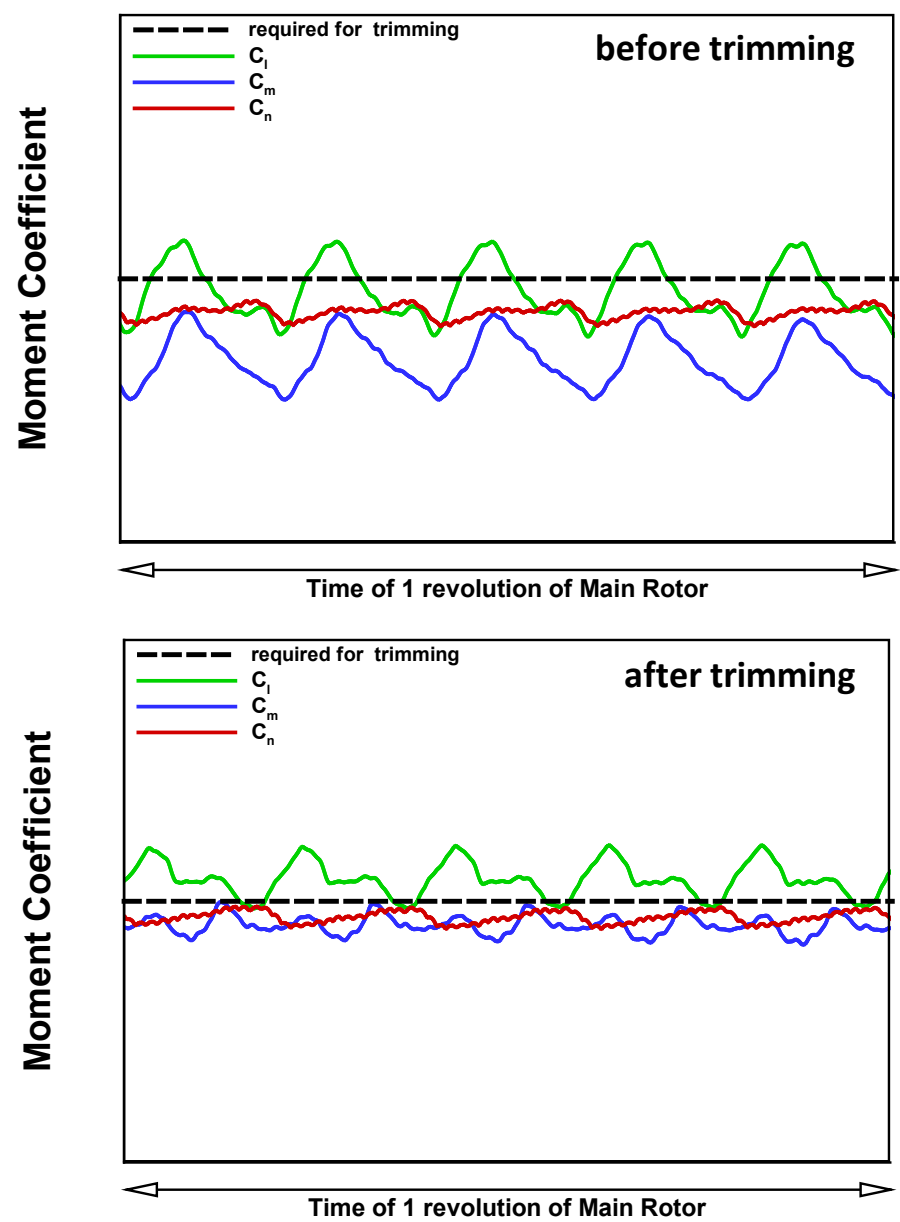

Fig. 6 Coefficients of global moments $\left(\mathrm{Cl}, \mathrm{C}_{m}, C_{n}\right)$ acting on the helicopter vs. time, before (top) and after (bottom) trimming procedure

Analysing Fig. 5 and Fig. 6 one may notice that even after first step of the helicopter trimming, the resultant forces and moments are oscillating around zero (which is the goal of the trimming) much more exactly than it has place in the case before trimming. In particular, the balancing of aerodynamic lift and helicopter weight is improved through trimming by the factor 5 .

Figure 7 presents qualitative results of simulation of trimmed hover flight of the helicopter: static pressure contours visualised on a helicopter surface.

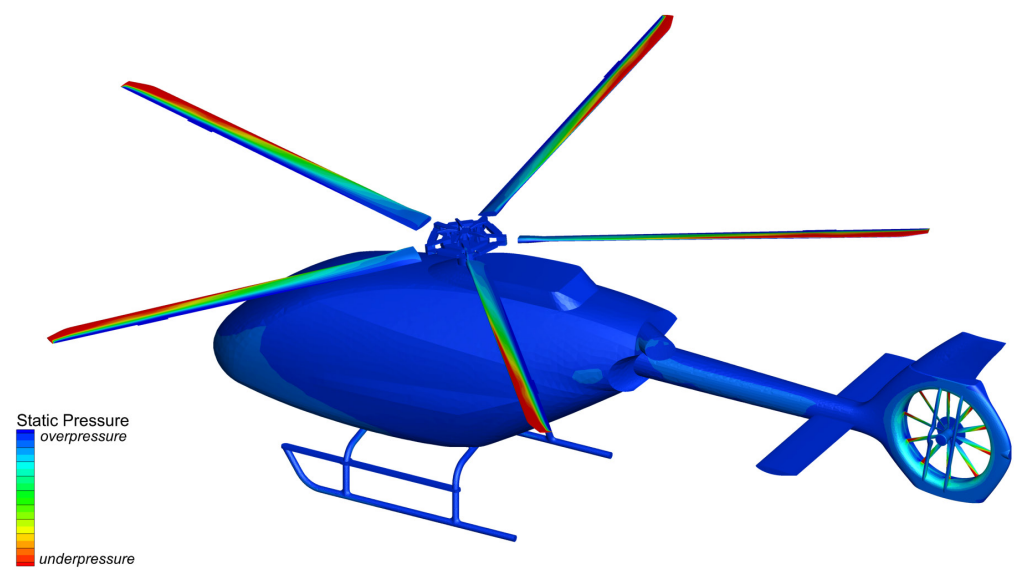

Fig. 7. Results of simulation of fully balanced hover of the helicopter. Static-pressure contours visualised on a helicopter surface 


\section{Conclusions}

The advanced methodology of computational simulation of a helicopter in trimmed flight has been developed and implemented. The methodology is based on the solution of URANS equations in a domain surrounding the flying helicopter. The URANS solver is coupled with the solver determining a main-rotor-blade flapping. The helicopter trimming procedure is based on results of several simulations of helicopter flight conducted using also the URANS-solution approach. Preliminary tests of the presented methodology confirm its high potential and suitability in rotorcraft research and development.

\section{References}

[1] Inc., ANSYS FLUENT User's Guide. Release 18.5. Available from: http://www.ansys.com.

[2] Peters, D. D., Barwey, D., A general theory of rotorcraft trim, Mathematical Problems in Engineering, Vol. 2, Is. 1, pp. 1-34, 1996.

[3] Peters, D. A., Chouchane, M., Fulton, M., Helicopter trim with flap-lag-torsion and stall by an optimized controller, Journal of Guidance, Control, and Dynamics, Vol. 13, No. 5, pp. 824-834, 1990.

[4] Hassan, H. S., Bayoumy, A. M., El-Bayoumi, G. M., Abdelrahman, M. M., Modelling, Trimming and Simulation of a Full-Scale Helicopter, $7^{\text {th }}$ International Conference on Aerospace Sciences \& Aviation Technology, ASAT-17, Cairo, Egypt 2017. 